Dirk G. Baur ${ }^{1}$ Thomas Dimpfl²

\title{
Think again: volatility asymmetry and volatility persistence
}

\author{
${ }^{1}$ UWA Business School (Accounting and Finance), The University of Western Australia (M251), 35 Stirling Highway, Crawley, \\ WA6009, Australia,E-mail: dirk.baur@uwa.edu.au \\ ${ }^{2}$ University of Tübingen, Tübingen, Germany. https://orcid.org/0000-0003-3415-7412.
}

\begin{abstract}
:
We use a leveraged quantile heterogeneous autoregressive model of realized volatility to illustrate that volatility persistence and the asymmetric "leverage" effect are high volatility phenomena. More specifically, we find that (i) low volatility is not persistent, but high volatility all the more, even featuring properties of explosive processes; and (ii) asymmetry of volatility is only a high volatility phenomenon and there is no asymmetry in low volatility regimes. Our results turn out to be robust to the choice of the realized variance estimator, in particular with respect to jumps. The analysis illustrates that quantile regression can provide information that is hidden in commonly used GARCH or realized volatility models. The quantile regression results can also be linked to the weak empirical evidence of the leverage effect and the volatility feedback effect.
\end{abstract}

Keywords: quantile regression, realized volatility, volatility asymmetry, volatility feedback, volatility persistence DOI: $10.1515 /$ snde-2017-0020

\section{Introduction}

Asymmetric volatility is a well-documented empirical phenomenon which states that the conditional volatility is negatively related to returns (see, inter alia, Campbell \& Hentschel, 1992; Engle \& Ng, 1993; French, Schwert \& Stambaugh, 1987). Therefore, a number of econometric models have been developed that allow to differentiate between the impact of positive and negative returns on the conditional future volatility. The asymmetric GARCH models of Nelson (1991) and Glosten, Jagannathan, and Runkle (1993) are the most prominent examples.

In what follows we propose to use quantile regression to shed additional light on the asymmetric volatility phenomenon. We find that volatility is in general not asymmetric with respect to positive or negative shocks with the exception of high volatility phases. Furthermore, the persistence of volatility also depends on the order of magnitude of the volatility, i.e. the volatility regime. This is information that cannot be unveiled in linear models that consider the expected value of a random variable only, in contrast to quantile regressions which model the complete conditional distribution of that random variable. Lastly, we document that jumps do not affect the detection of persistence in our empirical application. To investigate the effect of jumps in more detail, we draw on a simulation study.

Our findings are directly related to the literature on asymmetric volatility and the leverage effect. Bekaert and $\mathrm{Wu}(2000)$ provide an overview of the early literature. Recently, in particular with the availability of high frequency data, more sophisticated models are estimated. Ederington and Guan (2010) consider the impact of equally large positive and negative return shocks. They find that negative shocks are followed by an increase in volatility. However, the effect of positive return shocks seems model-dependent: in the asymmetric GARCH models, volatility rises slightly while implied and realized volatilities decline. Bekaert, Engstrom, and Ermolov (2015) propose a model that allows for two regimes of shocks (labeled good and bad environment) and a conditional variance forecast based on a good or a bad environment. Patton and Sheppard (2015) also distinguish between good and bad volatility by decomposing realized volatility using signed returns and find that negative returns are much more important in predicting volatility than positive returns. Linton, Whang, and Yen (2016) develop a test for this asymmetry that is based on the conditional distribution of volatility but do not find strong support for the hypothesis that negative returns are associated with larger volatility. We contribute to this literature by using a quantile regression framework to analyze good (low) and bad (high) volatility regimes.

Similar to our study, Badshah (2013) uses quantile regression to estimate the impact of positive and negative returns on changes of volatility indices whilst this paper focuses on the level of volatility and estimates its

Dirk C. Baur is the corresponding author.

(C)2019 Walter de Gruyter $\mathrm{CmbH}$, Berlin/Boston.

This content is free. 
quantile-dependent dynamics in the framework of what we will call the leveraged quantile heterogeneous autoregressive (LQHAR) model which is based on the work of Corsi and Renò (2012). In contrast, Baur and Dimpfl (2016) illustrate how a quantile autoregression model of returns can be used to estimate volatility and identify asymmetric effects between positive and negative returns on future volatility. Finally, $\mathrm{Wu}$ (2001) derives and estimates a model for the log price-dividend ratio which is capable of accounting for leverage and volatility feedback effects. He finds that both of these are important determinants in explaining asymmetric volatility. By construction, however, the model is not able to account for the level of volatility.

The results of the quantile regression also have implications for leverage and the volatility feedback effect. Based on our findings, both phenomena only exist in high volatility regimes. Economically, this result is not surprising. If volatility is low, a change in a firm's leverage should matter less than if volatility is high. Similarly, increased volatility should have a stronger feedback effect on returns and, in turn, volatility if volatility is high than if volatility is low. The findings are consistent with recent studies that provide support for the conditional effects, e.g. only in bear markets. Talpsepp and Rieger (2010) find that economic development and market capitalization as a share of GDP are the most important drivers for volatility asymmetry. Leverage and short selling are found to increase volatility in bear markets only. Haas et al. (2013) also find evidence that leverage affects volatility only in bear markets. Park (2011) considers asymmetry in the volatility of major exchange rates and finds that asymmetric herding provides a better explanation than the leverage and the volatility feedback effect.

The paper proceeds as follows. Section 2 presents the econometric model and describes the data. Section 3 reports and discusses the estimation results of the quantile-dependent volatility dynamics including asymmetric volatility effects. In Section 4 we consider the (asymmetric) volatility feedback effect in more detail. Section 5 evaluates the robustness of our results, in particular assessing the impact of jumps in the estimation of persistence using a simulation study. Section 6 summarizes the main results and concludes.

\section{Methodology and data}

\subsection{The asymmetric LQHAR model}

The leverage and the volatility feedback effect have so far been analyzed to the greatest extent based on discrete time models, using in particular GARCH models. In the continuous time context, Corsi and Renò (2012) recently introduced the leveraged HAR model, stating that when modeling realized volatility, it is necessary to mimic the properties and dynamics of the assumed quadratic variation. The LHAR model for realized variance (which is one out of three proposed specifications) reads as

$$
R V_{t}=c+\beta_{1} R V_{t-1}+\beta_{2} R V_{t-1}^{w}+\beta_{3} R V_{t-1}^{m}+\beta_{3} r_{t-1}^{2} d_{t-1}+\varepsilon_{t},
$$

where $r_{t}$ is the close-to-close return and $d_{t}$ is a dummy variable which is one if $r_{t-1}<0$ and zero otherwise. The weekly (monthly) variance $R V_{t}^{w}\left(R V_{t}^{m}\right)$ is calculated as the average of daily variances $R V_{t}$ from $t-4(t-21)$ to $t$. $\varepsilon_{t}$ is an i.i.d. white noise process. While Corsi and Renò (2012) design the model to deliver optimal forecasts of $R V_{t+h}$, our focus is on learning about the dependence structure of the volatility across the entire distribution of the realized variance and we propose to use the LHAR model as a starting point.

The quantile regression framework introduced by Koenker and Bassett (1978) provides the tools to model the conditional distribution of a random variable. In the present context, the extension to quantile autoregressive (QAR) models of Koenker and Xiao (2006) allows us to characterize the dependence structure of realized volatility across its distribution. We follow Andersen et al. (2003) and treat the realized variance as an observed time series of variance observations which constitute the object of further interest. QAR in general extends the possibilities to describe the features of a time series beyond a standard autoregressive model. The general QAR $(p)$ model for a time series variable $y_{t}$ is

$$
Q_{y_{t}}\left(\tau \mid y_{t-1}, \ldots, y_{t-p}\right)=\theta_{0}(\tau)+\theta_{1}(\tau) y_{t-1}+\ldots+\theta_{p}(\tau) y_{t-p},
$$

where $\tau$ denotes the quantile of interest with $\tau \in(0,1)$ and $\theta_{i}(\tau)$ are quantile specific parameters. The model is particularly useful if the properties of the time series process depend on the level of the time series. As studies by Baur, Dimpfl, and Jung (2012) and Zhu et al. (2016), or Zhu et al. (2015) showed, the dynamic properties of time series are indeed quantile dependent and this also holds for volatility processes (cp. Badshah, 2013).

Combining the LHAR model with the quantile autoregressive estimation approach leads to the central model of our analysis, the leveraged quantile heterogeneous autoregressive (LQHAR) model: 


$$
\begin{aligned}
Q_{R V_{t}}(\tau)= & \theta_{0}(\tau)+\theta_{1}(\tau) r_{t-1}^{2}+\theta_{2}(\tau) r_{t-1}^{2} d_{t-1} \\
& +\theta_{3}(\tau) R V_{t-1}+\theta_{4}(\tau) R V_{t-1}^{w}+\theta_{5}(\tau) R V_{t-1}^{m} .
\end{aligned}
$$

In contrast to the LHAR model in Equation (1), we add the additional regressor $r_{t-1}^{2}$. The reason is that it provides additional information related to the entire trading day which is by construction not captured in $R V_{t-1}$. For example, if $r_{t}$ is (close to) zero, $R V_{t}$ might be high or low, depending on the trading pattern throughout the day. If prices changed heavily and returned to their initial value at the closing of the market, $R V_{t}$ will be high.

From Equation 2 it is now possible to deduce the quantile dependent structure of volatility asymmetry and volatility persistence. The asymmetric effect of past returns on RV is directly observed through the estimation of $\theta_{1}$ and $\theta_{2}$. Persistence is slightly more complicated. Of course, the parameter $\theta_{3}$ is of great interest as it provides a first impression of the immediate persistence. In the long run, however, it is necessary to consider the full dynamics as captured by $\theta_{3}, \theta_{4}$, and $\theta_{5}$. In the standard HAR specification of Corsi (2009), the weekly and monthly aggregates replace a longer autoregressive dynamic. In the end, the specification in Equation 1 is a short form of an $\mathrm{AR}(22)$ :

$$
y_{t}=\alpha_{1} y_{t-1}+\alpha_{2} y_{t-2}+\ldots+\alpha_{22} y_{t-22}+\varepsilon_{t} .
$$

Now for this general AR(p) process to be stationary, a necessary (albeit not sufficient) condition is that the sum of the autoregressive parameters be lesser than 1 in absolute value, i.e. $\left|\sum_{i=1}^{22} \alpha_{i}\right|<1$. In the cascading structure of the HAR, these 22 coefficients are summarized in 3 coefficients which are related to the AR(p) as follows: $\alpha_{1}=\beta_{1}+\frac{1}{5} \beta_{2}+\frac{1}{22} \beta_{3}$ etc. Basically the HAR imposes the restriction that $\alpha_{2}$ to $\alpha_{5}$ and $\alpha_{6}$ to $\alpha_{22}$ are identical. Hence, in the HAR context, the stationarity condition can easily be stated as $\left|\beta_{1}+\beta_{2}+\beta_{3}\right|<1$.

In the QAR model, Koenker and Xiao (2006) show that a process that is globally stationary might exhibit explosive behavior in the tails of the distribution. For the process to be stationary, the expected value of the QAR parameters needs to be lesser than one in absolute value. In our LQHAR model, the stationarity condition is, thus, that the expected value of the sum $\left(\theta_{3}(\tau)+\theta_{4}(\tau)+\theta_{5}(\tau)\right)$ is lesser than one. We can thus sum the coefficients $\left(\theta_{3}(\tau)+\theta_{4}(\tau)+\theta_{5}(\tau)\right)$ for each $\tau$ to obtain a measure for the overall persistence in the LQHAR model. The stationarity condition is satisfied if, in addition, the absolute value of the sum over $\tau$ is lesser 1 .

It should be noted that the model presented in Equation (2) has a structure which is similar to the threshold GARCH model of Glosten, Jagannathan, and Runkle (1993). The heterogeneous terms account for the long persistence which is typically found in realized variance time series, but also a typical feature of the GARCH model where it is captured by the GARCH term. The dependence on lagged squared returns is similar to the ARCH term in the GARCH model (depending on the exact specification of the mean model of course). In order to highlight the gains in information we consider two benchmark models to compare the QAR model to. First, the linear LHAR model of Corsi and Renò (2012) which is parametrized as our QAR model given in Equation (2) and, hence, reads as follows:

$$
\begin{aligned}
R V_{t}= & \beta_{0}+\beta_{1} r_{t-1}^{2}+\beta_{2} r_{t-1}^{2} d_{t-1}+\beta_{3} R V_{t-1} \\
& +\beta_{3} R V_{t-1}+\beta_{4} R V_{t-1}^{w}+\beta_{5} R V_{t-1}^{m}+\varepsilon_{t}
\end{aligned}
$$

where $\varepsilon_{t}$ is an i.i.d. white noise process. The second benchmark model is the threshold GARCH model of Glosten, Jagannathan, and Runkle (1993) which is specified as follows:

$$
\begin{gathered}
r_{t}=\mu+\beta r_{t-1}+u_{t}, \quad u_{t} \sim N\left(0, h_{t}\right) \\
h_{t}=\omega+\alpha u_{t-1}^{2}+\gamma h_{t-1}+\delta I\left(u_{t-1}<0\right) u_{t-1}^{2},
\end{gathered}
$$

where $r_{t}$ is the close-to-close return and $I(\cdot)$ is the indicator function that takes on a value of 1 if $\varepsilon_{t-1}<0$ and 0 otherwise. The main parameter of interest is then $\delta$ which captures the asymmetric response of the variance $h_{t}$ to lagged innovations. We use an AR(1) process for the mean equation in order to account for market imperfections (cp. Campbell \& Shiller, 1988).

A drawback of our specification is that we include the correlated variables $r_{t}^{2}$ and $R V_{t}$ at the same time in our models. The average correlation observed in our data is 0.51 . We therefore also estimate a reduced form of Equation (2) where we drop $r_{t-1}^{2}$ and replace $r_{t-1}^{2} d_{t-1}$ by $R V_{t-1} d_{t-1}$ (labeled "robustness regression model"). This allows us to test and ensure that the high correlation does not bias our results. On the other hand, we lose the interpretation of the daily shock which is the driving force in the GARCH model, and, thus, direct comparability of these models. 


\subsection{Data}

Our models build on realized variances which have been put forward by Andersen et al. (2003) as an estimator for the quadratic variation of the assumed underlying continuous-time price process. The price process is generally assumed to consist of a continuous sample path and a jump component. The standard realized variance estimator based on squared returns over five minute intervals includes the variance contributions of both jumps and the continuous component (cp. Barndorff-Nielsen \& Shephard, 2004). Thus, in particular large RV observations might include a jump component. As jumps are assumed to be not persistent, this might impact on our dependence analysis. We therefore also consider the MedRV estimator of continuous volatility which is robust to jumps (for details see Shephard \& Sheppard, 2009).

The data used in our analysis are obtained from the Oxford-Man Institute of Quantitative Finance's realized library (Heber et al., 2009) from January 03, 2000 (or later, depending on the index) to November 30, 2016. In addition to the two realized variance time series $R V$ (calculated on 5 minute intervals) and $M e d R V$ we also use close-to-close log-returns $r_{t}$ (in percent) for the following 20 stock market indices: AEX, AORD, Bovespa, CAC40, DAX, DJIA, EuroStoxx50, FTSE, FTSEMIB, FTSTI, HSI, IBEX, IPC Mexico, KS11, NASDAQ, Nikkei 225, Russel 2000, S\&P CNX Nifty, S\&P 500, and SSMI. The individual samples differ with respect to the number of observations, ranging from 4310 observations of the CAC40 to 3651 observations of the S\&P CNX Nifty index. All time series are stationary [based on the results of an augmented Dickey-Fuller test with number of lags selected by the Schwarz (1978) Bayesian information criterion].

Figure 1 presents time series plots and a correlation diagram for a representative entity of our sample, namely the S\&P 500. The time series of returns (depicted in gray in the upper graph) exhibits the welldocumented volatility clustering, in particular at the beginning of the 21st century and during the financial crisis from mid 2007 to mid 2009. This is supported by the second graph (which depicts realized volatility $R V_{t}$ ) where phases of higher and lower volatility seem to alternate. The last graph presents daily cross-correlations for the return and the variance as $\operatorname{corr}\left(R V_{t}, r_{t+j}\right)$ for $j \in[-30 ; 30]$. The vertical lines span a $95 \%$ confidence band which is based on GMM methodology using a Bartlett kernel with two lags to account for serial correlation during the estimation of the GMM residual variance-covariance matrix. As can be seen, the contemporaneous correlation is negative which is in line with previous studies on the volatility feedback effect (for example Bollerslev, Litvinova \& Tauchen, 2006). The cross-correlations also indicate the presence of an extensive negative feedback effect of volatility on future returns. 

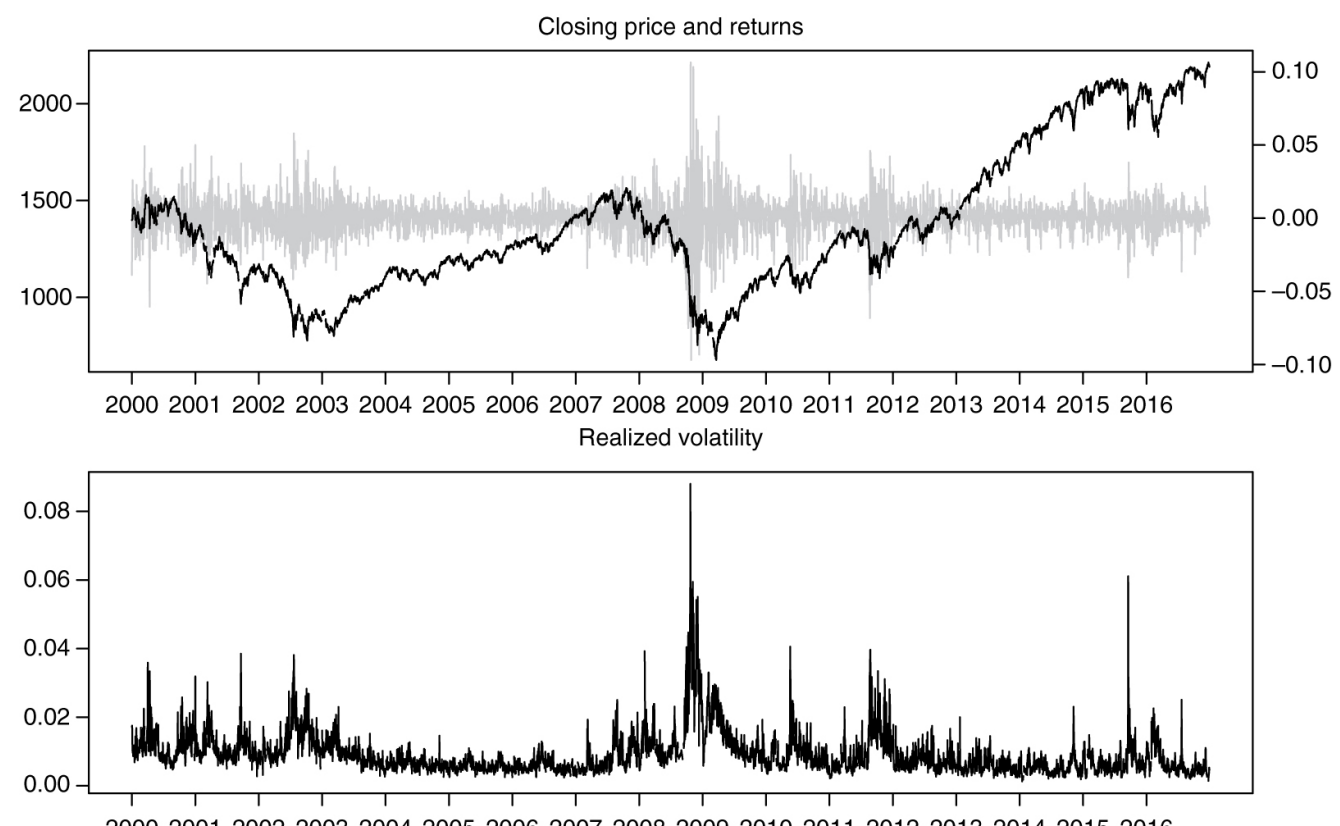

20002001200220032004200520062007200820092010201120122013201420152016 Daily cross-correlation

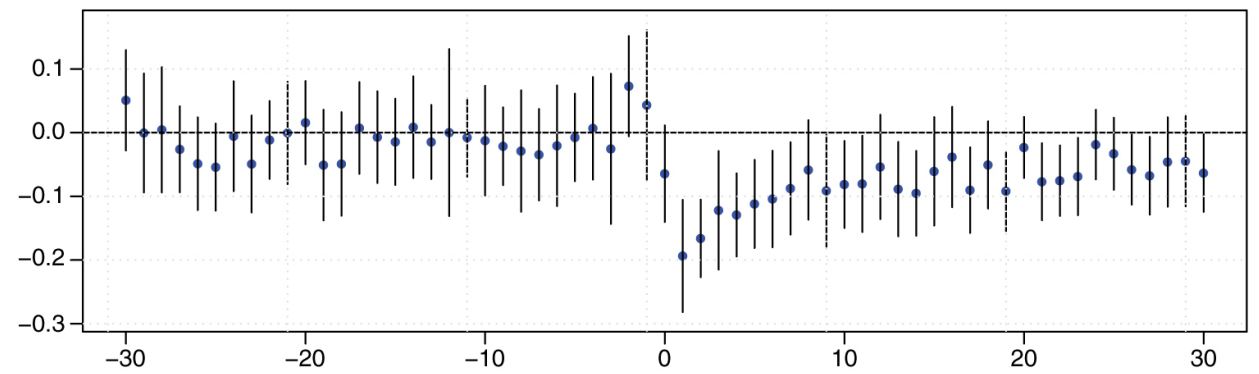

Figure 1: Time series plots and correlations.

The figures present time series plots and correlations for the S\&P 500 data. The upper graph holds closing prices (black) and returns (gray). The middle graph presents realized volatility and the lower graph presents cross-correlations for the return and the variance as $\operatorname{corr}\left(R V_{t}, r_{t+j}\right)$ together with a $95 \%$ confidence interval (vertical lines). The necessary standard errors are based on GMM methodology using a Bartlett kernel with two lags to account for serial correlation during the estimation of the GMM residual variance-covariance matrix.

\section{Quantile-dependent volatility dynamics}

The model is implemented for a discrete choice of quantiles $\tau \in\{0.01,0.02, \ldots, 0.99\}$. Figure 2 graphically presents the estimated coefficients of the quantile autoregression model given in Equation (2) for all stock indices across all considered quantiles. The effect of squared lagged returns as well as the effect of the squared negative returns is essentially stable for low quantiles of the distribution of realized variance, i.e. for low variances. However, the impact becomes more important in high volatility phases as the order of magnitude of $\hat{\theta}_{1}$ and $\hat{\theta}_{2}$ increases markedly for the high quantiles between 0.8 and 0.99 . It turns out that negative shocks tend to increase the variance by more (as $\hat{\theta}_{1}+\hat{\theta}_{2}>0$ ) than positive shocks in these quantiles. This suggests that overnight valuation insecurity plays a non-negligible role in the explanation of the (intra-)daily realized variance. 

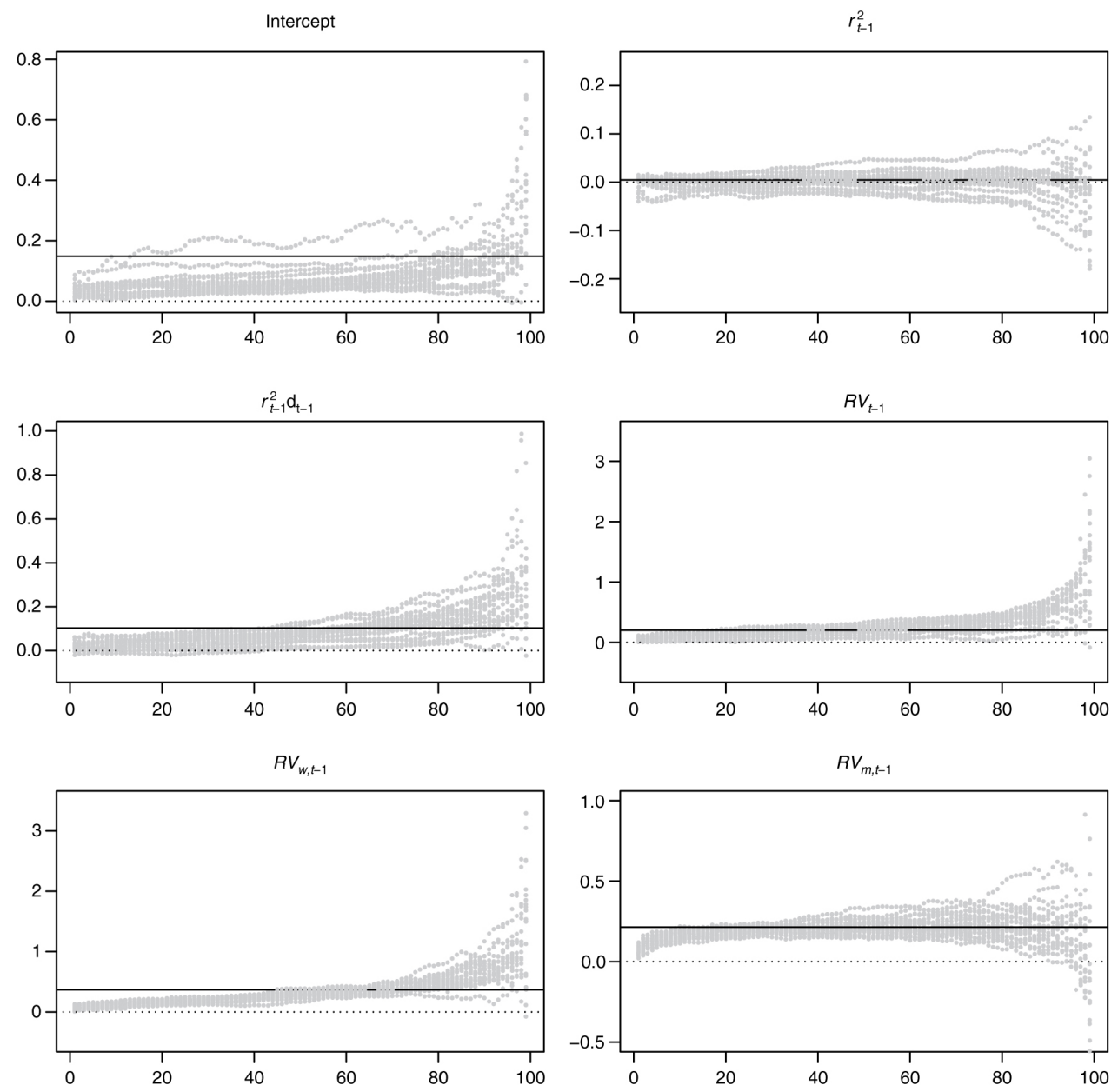

Figure 2: Quantile model estimates based on RV.

The graphs depict the coefficient estimates of the quantile autoregressive model for realized variance for the 20 reference entities (gray). The black solid line is the average of the respective estimates in a linear regression model.

Our results highlight the well-documented variance persistence, but we can draw even finer conclusions from the three bottom right graphs in Figure 2. The estimates of $\theta_{3}, \theta_{4}$ and $\theta_{5}$ are rather small and well below one in low quantiles. However, they gradually increase when moving to higher quantiles. As low quantiles are associated with low variances while high quantiles belong to high variances, the plot implies that variance persistence is actually a question of the level of the variance. If the variance is low, the persistence is rather weak. In contrast, if the variance is high, the persistence is high. For quantiles $\tau>0.9$ we find estimates of $\theta_{3}$ that exceed 1 which implies that upper quantiles exhibit even explosive, non-stationary behavior. This implies that extremely high variance is not only self-sustaining, but there is a self-nurturing effect that is in theory able to drive the variance to infinity. However, this only affects the uppermost tail of the variance distribution. The picture is similar for the impact of the weekly variance measures in that low quantiles are less susceptible to variance persistence as higher ones. Only the monthly variance exhibits a flatter pattern of coefficient estimates. They are close to zero for very low quantiles, rise to roughly 0.2 on average and remain there for the remaining quantiles. For the highest quantiles the effect is not clear-cut. Taken together, we find a total persistence effect as presented in Figure 3. 


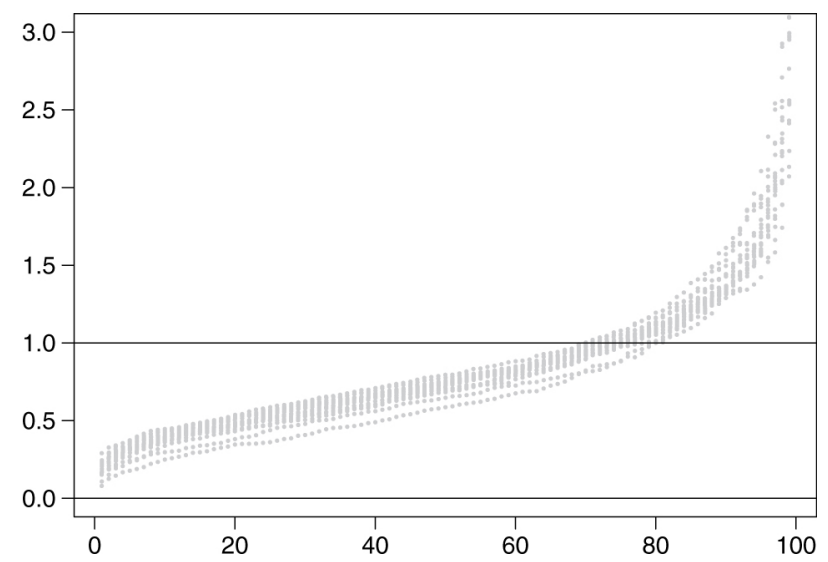

Figure 3: Persistence in the quantile model.

The graph displays the sum of the coefficient estimates $\hat{\theta}_{3}, \hat{\theta}_{4}$ and $\hat{\theta}_{5}$ for each $\tau$ for the 20 reference entities. Estimation is based on the full model using RV.

The sum of the coefficient estimates $\hat{\theta}_{3}, \hat{\theta}_{4}$, and $\hat{\theta}_{5}$ as presented in Figure 3 highlights the strong persistence of realized variance. From $\tau=0.75$ the average of the documented sum becomes larger than 1 , implying that the variance exhibits explosive behavior in the upper tail quantiles while still being stationary in a "classical" sense. The sum of the coefficients also highlights that the total persistence of the variance process is quite similar across all observed entities while the individual effects as documented in Figure 2 deviate more strongly.

Changing persistence, in particular, is an effect that cannot be deduced by any of our benchmark models. The solid lines in Figure 2 depict the OLS estimates based on Equation (3). The averages of $\hat{\beta}_{3}, \hat{\beta}_{4}$ and $\hat{\beta}_{5}$ across all stock indices are $0.202,0.368$, and 0.214 , respectively. While the size of the estimates is remarkable in an autoregressive framework, their sum is 0.784 and, thus, still far from implying the almost non-stationary variance process as documented in the subsequent GARCH model.

Table 1 holds the estimation results for the TGARCH model of Glosten, Jagannathan, and Runkle (1993). The persistence parameter $\gamma$ is 0.9 on average while the sum $\alpha+\gamma+\frac{1}{2} \delta$ is 0.98 on average which is just slightly below 1 , the value which would indicate non-stationarity of the variance process. Of course the implemented constraints prevent estimates that are not compatible with a stationary variance process during the estimation. Indeed, our quantile regression results suggest that there are parts of the distribution of realized variance that are modeled best when allowing a non-stationary behavior.

\section{Table 1: Benchmark estimation.}

\begin{tabular}{lrrrrrr}
\hline Stock & $\mu$ & $\beta$ & $\omega(\mathbf{1 0 0 0})$ & $\alpha$ & $\gamma$ & $\delta$ \\
\hline DAX & -0.0001 & -0.0090 & $0.0021^{* * * *}$ & 0.0014 & $0.9135^{* * * *}$ & $0.1369^{* * *}$ \\
FTSE & $-0.0004^{* * *}$ & $-0.0358^{* *}$ & 0.0007 & 0.0220 & $0.9114^{* * *}$ & $0.1137^{* * *}$ \\
IBEX & -0.0002 & -0.0087 & 0.0016 & 0.0106 & $0.9214^{* * *}$ & $0.1111^{* * *}$ \\
S\&P 500 & 0.0001 & $-0.0524^{* * *}$ & $0.0021^{* * *}$ & 0.0000 & $0.8908^{* * *}$ & $0.1765^{* * *}$ \\
DJIA & $0.0002^{* *}$ & $-0.0498^{* * *}$ & $0.0018^{* * *}$ & 0.0000 & $0.8922^{* * *}$ & $0.1790^{* * *}$ \\
EuroStoxx50 & -0.0002 & $-0.0440^{* * *}$ & 0.0024 & 0.0000 & $0.9032^{* * *}$ & $0.1636^{* * *}$ \\
AEX & $-0.0003^{* *}$ & $-0.0294^{*}$ & $0.0013^{*}$ & 0.0000 & $0.9160^{* * *}$ & $0.1401^{* * *}$ \\
AORD & 0.0001 & -0.0176 & 0.0010 & 0.0080 & $0.9106^{* * *}$ & $0.1206^{* * *}$ \\
Bovespa & -0.0001 & -0.0025 & $0.0051^{* * *}$ & $0.0088^{* * *}$ & $0.9289^{* * *}$ & $0.0871^{* * *}$ \\
CAC40 & -0.0002 & $-0.0539^{* * *}$ & 0.0019 & 0.0000 & $0.9134^{* * *}$ & $0.1410^{* * *}$ \\
FTSEMIB & $-0.0004^{* * *}$ & $-0.0664^{* * *}$ & 0.0014 & 0.0160 & $0.9180^{* * *}$ & $0.1110^{* * *}$ \\
FTSTI & $-0.0003^{* * *}$ & $-0.0358^{* *}$ & 0.0006 & $0.0693^{* *}$ & $0.8948^{* * *}$ & $0.0595^{* * *}$ \\
HSI & $-0.0004^{* * *}$ & $-0.0376^{* *}$ & 0.0011 & 0.0349 & $0.9330^{* * *}$ & $0.0368^{* * *}$ \\
NASDAQ & -0.0000 & $-0.0338^{* *}$ & 0.0014 & 0.0165 & $0.9142^{* * *}$ & $0.1121^{* * *}$ \\
KS11 & $-0.0005^{* * *}$ & $-0.0375^{* *}$ & 0.0011 & 0.0523 & $0.9103^{* * *}$ & $0.0587^{* * *}$ \\
IPC Mexico & $0.0003^{* *}$ & $0.0775^{* * *}$ & $0.0020^{* *}$ & $0.0117^{* *}$ & $0.9156^{* * *}$ & $0.1200^{* * *}$ \\
Nikkei 225 & $-0.0004^{* * *}$ & $-0.0528^{* * *}$ & $0.0045^{* * *}$ & $0.0620^{* * *}$ & $0.8550^{* * * *}$ & $0.0974^{* * *}$ \\
S\&P CNX Nifty & $0.0003^{*}$ & $0.0562^{* * *}$ & $0.0040^{* * *}$ & $0.0521^{* * *}$ & $0.8569^{* * *}$ & $0.1243^{* * *}$ \\
Russel 2000 & 0.0000 & -0.0255 & $0.0034^{* * *}$ & 0.0018 & $0.9090^{* * * *}$ & $0.1329^{* * * *}$ \\
SSMI & -0.0002 & -0.0206 & $0.0018^{* * * *}$ & $0.0450^{* * *}$ & $0.8782^{* * *}$ & $0.1149^{* * *}$ \\
Average & -0.0001 & -0.0240 & 0.0021 & 0.0206 & 0.9043 & 0.1169 \\
\hline
\end{tabular}


The table reports the estimation results of the asymmetric GARCH benchmark model Glosten, Jagannathan, and Runkle (1993) which is specified as

$r_{t}=\mu+\beta r_{t-1}+u_{t}, \quad u_{t} \sim N\left(0, h_{t}\right)$

$h_{t}=\omega+\alpha u_{t-1}^{2}+\gamma h_{t-1}+\delta I\left(u_{t-1}<0\right) u_{t-1}^{2}$,

where $I(\cdot)$ is the indicator function. ${ }^{* *}\left({ }^{* *},{ }^{*}\right)$ indicates significance on a $1 \%(5 \%, 10 \%)$ significance level. The estimator of $\omega$ was multiplied by 1000 to enhance readability.

While the results documented in Table 1 show that the variance is highly persistent, the models cannot differentiate between different levels of volatility and the conclusion would be an average persistence for all possible regimes of volatility. Different regimes of volatility can be identified in Figure 1 with a low volatility (tranquil) period after 2003 up to the outbreak of the global financial crisis in mid-2007 and a high volatility period during the global financial crisis. These regimes can be expected to exhibit different volatility dynamics which are hidden by the averaging implicitly performed in a GARCH model or a simple model for realized variance. ${ }^{1}$

To ensure that the results presented above are robust, we also estimate the robustness regression model which is a model for realized volatility only as described in Section 2.1. Figure 4 presents the coefficient estimates of the QAR model along with the OLS estimates. The results are qualitatively the same as the ones presented in Figure 2. In particular, the observation that the persistence is more pronounced for high quantiles, holds. Also, the persistence associated with negative returns is generally slightly higher than the overall average and increases in upper quantiles, i.e. high variance regimes. Looking at the sum of $\hat{\theta}_{1}, \hat{\theta}_{3}$, and $\hat{\theta}_{4}$ reveals that the sum is greater than 1 starting with $\tau=0.73$ which is comparable to the result using RV. While the persistence and stationary implications hold on average, Figure 5 reveals that the dispersion in the extreme upper quantiles is higher than in the previous model. The overall mean of all estimates is 0.844 , however, which still implies a pronounced persistence on average for the reference entities in our sample.
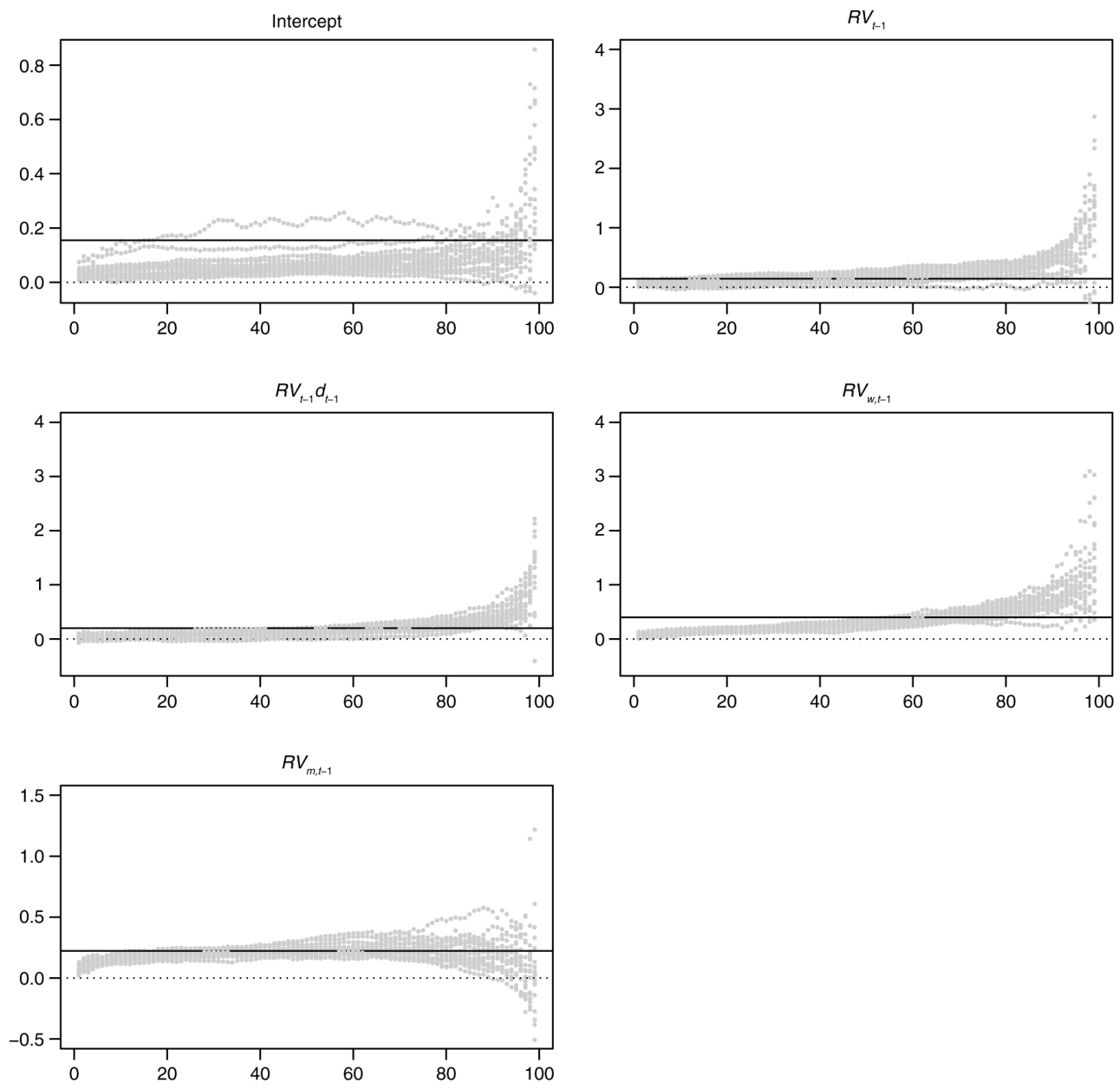

Figure 4: Robustness regression based on RV.

The graphs depict the coefficient estimates of the quantile autoregressive model for realized variance for the 20 reference entities (gray). The black solid line is the average of the respective estimates in a linear regression model. 


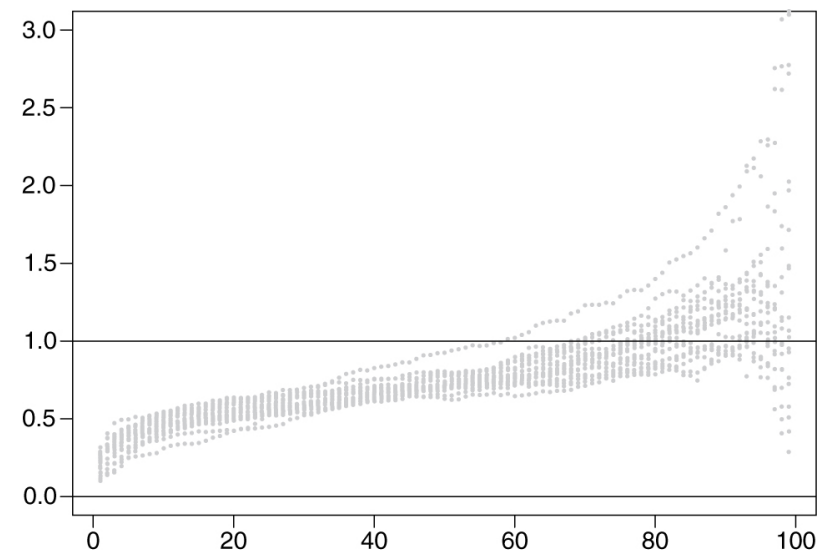

Figure 5: Persistence in the quantile model.

The graph displays the sum of the coefficient estimates $\hat{\theta}_{1}, \hat{\theta}_{3}$ and $\hat{\theta}_{4}$ for each $\tau$ for the 20 reference entities. Estimation is based on the robustness regression model using RV.

\subsection{The presence of jumps}

An issue in modeling the realized variance might be the presence of jumps. Realized variance as used in the foregoing analysis contains both continuous and jump variation. Therefore, days with higher variance are more likely to have experienced a jump than days with low variance. High variance, however, refers to the upper quantiles in the QAR model results as depicted in Figure 2. It might therefore be the case that the explosive behavior documented in Figure 2 is a result of the presence of jumps. On the other hand, if jumps dominate the price process in the upper quantiles, they might as well completely overshadow the persistence of the continuous price variation part in upper quantiles as jumps are assumed to be independent over time.

To address this issue, we repeat the estimation of the quantile autoregression model given in Equation (2) for the continuous variance $M e d R V$. This allows us to draw conclusions with respect to the persistence of volatility only and gives an idea on the effect of jumps. The estimation results for all stock indices across all considered quantiles are depicted in Figure 6. As can be seen, removing the jump variation does not qualitatively alter our main conclusions. In particular, the persistence documented for high quantiles is still clearly present: it has neither been removed nor has the autoregressive behavior in upper quantiles been dampened such that it would now be compatible with a stationary process. Hence, the use of RV which includes the jump component does not impact on the detection of persistence in high quantiles of the variance. The pattern of the sum of the coefficient estimates $\hat{\theta}_{4}, \hat{\theta}_{5}$, and $\hat{\theta}_{6}$ (not reported) is also similar to the pattern documented in Figure 3. 

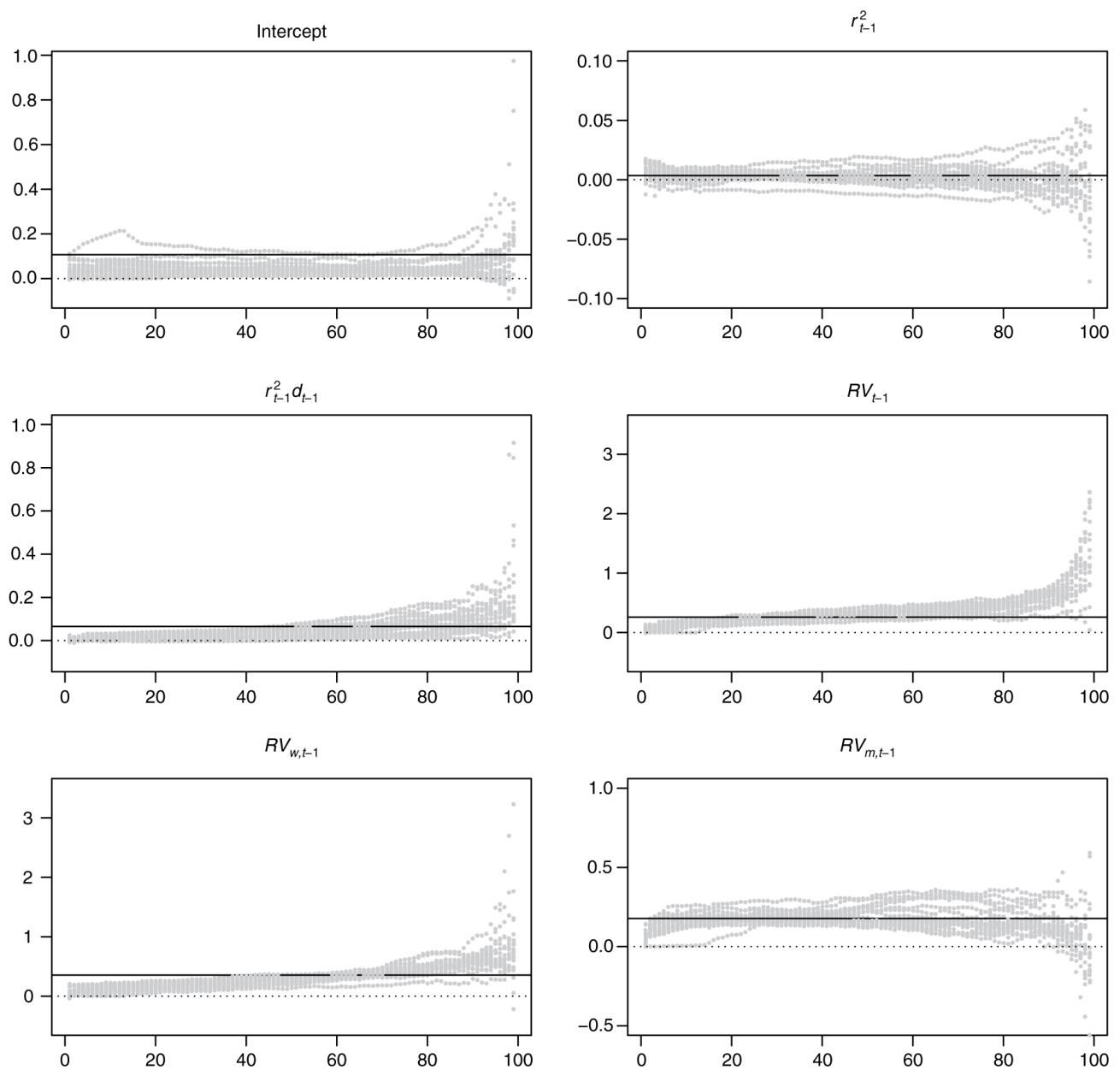

Figure 6: Quantile model estimates based on MedRV.

The graphs depict the coefficient estimates of the quantile autoregressive model for the continuous variation part in realized variance for the 20 reference entities (gray). The black solid line is the average of the respective estimates in a linear regression model.

As in the previous subsection, to check that our results are not influenced by the model specification and choice of regressors, we implement the reduced model without $r^{2}$ components. Figure 7 presents the respective estimates of the quantile and OLS regressions. Again, the results documented in the previous subsection and in particular in Figure 4 are confirmed by this robustness check. Similarly, the pattern of the sum of the coefficient estimates $\hat{\theta}_{1}, \hat{\theta}_{3}$, and $\hat{\theta}_{4}$ (not reported) is in line with the one presented in Figure 5. In particular the dispersion in the upper quantiles did not remarkably increase. 

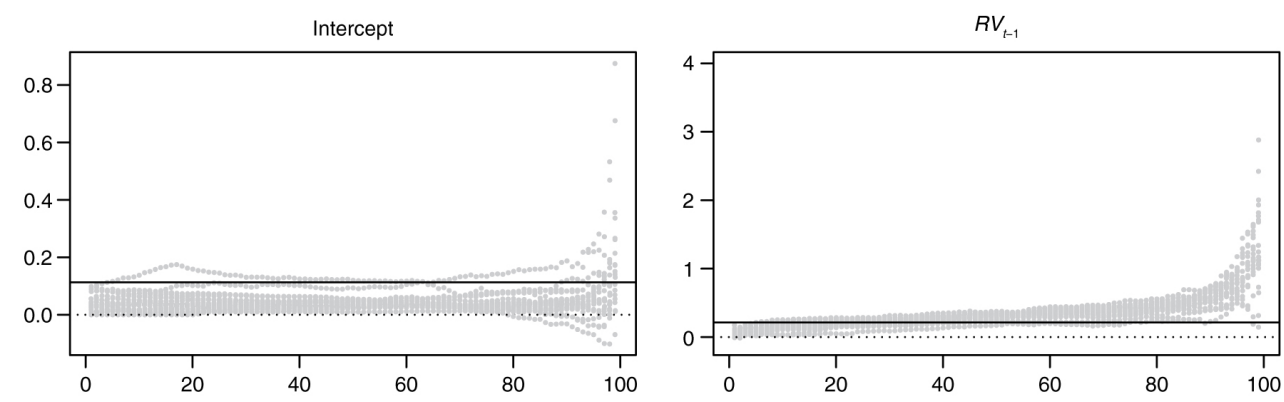

$R V_{t-1} d_{t-1}$
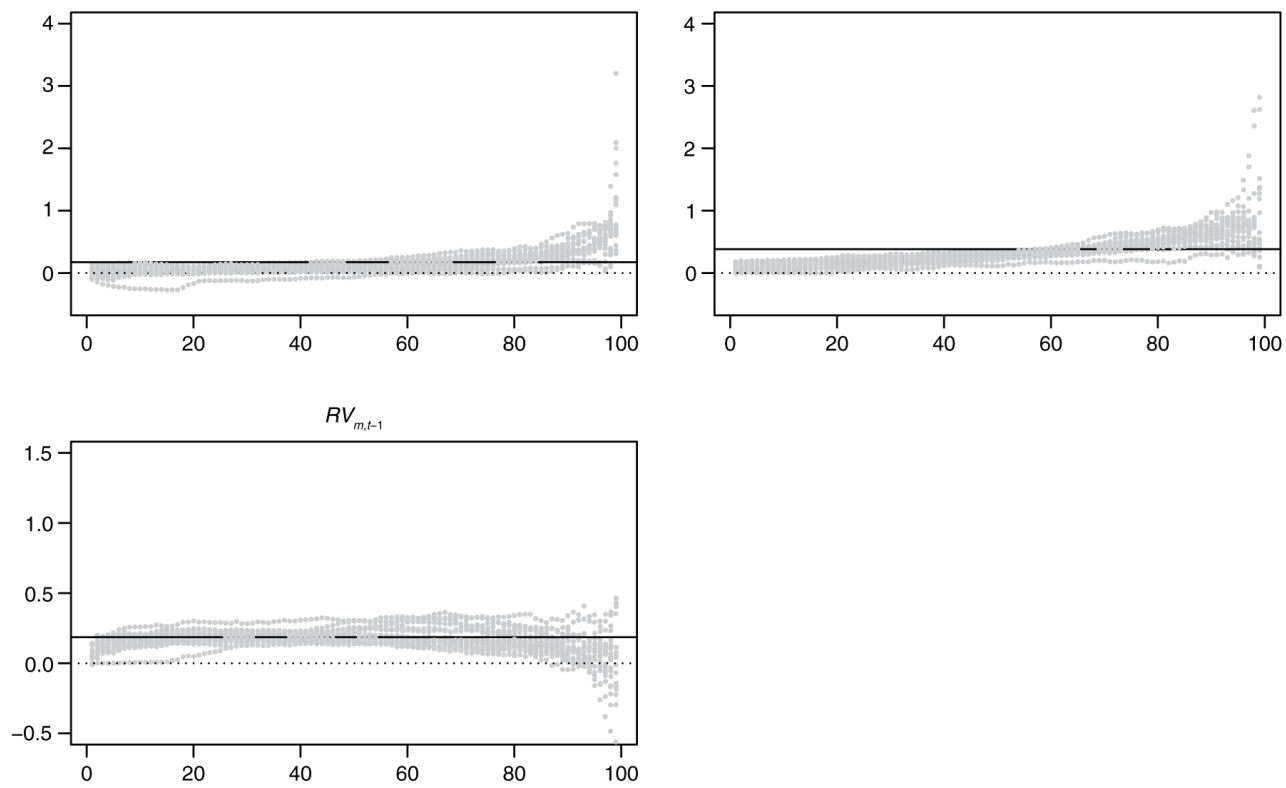

Figure 7: Robustness regression based on MedRV.

The graphs depict the coefficient estimates of the quantile autoregressive model for the continuous variation part in realized variance for the 20 reference entities (gray). The black solid line is the average of the respective estimates in a linear regression model.

\subsection{Conditional densities}

Our results also allow a finer differentiation with respect to the impact of lagged returns on the variance process. Again, in an asymmetric (threshold) GARCH model or in the linear realized variance model in Equation (3), the effect of the (lagged) return depends on the size and the sign of the return only, but it is independent of the level of the variance. Based on the quantile regression estimates we can compute the conditional density of the variance and thereby account for positive or negative returns and see how they influence the probability density function (pdf). We therefore construct a density forecast for a low (high) variance situation using the value at the $10 \%(90 \%)$ quantile of the empirical distribution of the variance and differentiate the effect of a negative and positive lagged return. For the latter we also use the $10 \%$ and $90 \%$ quantiles of the empirical return distribution. Plugging these values in the QAR function gives rise to the conditional densities as depicted in Figure 8. The two densities on the left (solid lines) present low variance regimes and the two densities on the right (dashed lines) are high variance regimes; blue (black) refers to positive (negative) lagged returns. 

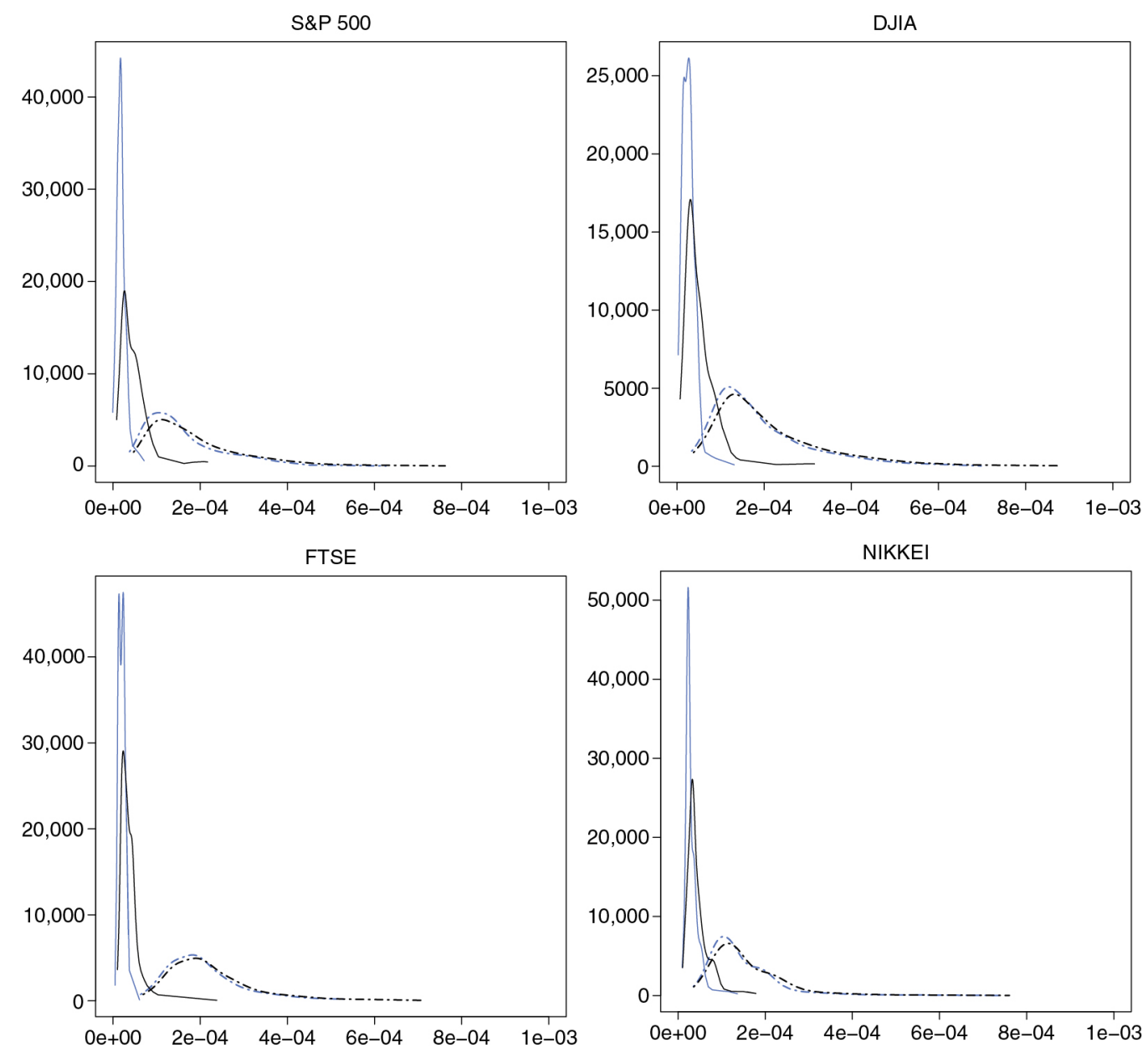

Figure 8: Density plots.

The graphs present density plots for four reference entities for different regimes. The left densities in each plot belong to a low volatility regime while the right densities depict a high volatility regime. A blue line refers to a positive return on the previous day while black depicts a negative return.

The graphs have two interesting implications. First, in a low volatility state, the pdf is much more condensed than it is in a high volatility state. Thus, irrespective of the lagged return, the dispersion of possible realizations of the variance is broader if the variance is high than when it is low. The second implication concerns the impact of lagged returns. In a low variance state, a negative lagged return shifts the distribution markedly to the right. This implies a higher expected variance if the lagged return is negative which is in line with the literature on asymmetric GARCH models. However, in a high variance regime, a lagged negative return has only a weak effect on the distribution of realized variance which is almost identical to an effect of a positive lagged return. It seems that in this situation the effect of $r_{t-1}$ is to some extent overshadowed by the high persistence of the variance itself.

Comparing Figure 2 and Figure 8 we have two results that seem inconsistent at first glance: Figure 2 implies that the asymmetric effect is more important in a high than in a low variance regime while the densities in Figure 8 suggest that in high variance regimes the effect of lagged returns does not depend on the sign as much. To solve this puzzle we have to consider the low volatility regime. As the variance is low (which can also be seen from the intercept plot in Figure 2), the effect of the lagged return is comparatively more important than in a high variance regime. Thus, even a small additional impact of the negative return as opposed to the positive return is able to shift the distribution considerably to the right. In a high state, it would have an even more pronounced effect, but it is eclipsed by the extremely high persistence of the variance itself. In other words, a negative return shock of the same size is in absolute terms less important in a low volatility state than in a high volatility state, while in relative terms its impact is more important in a low volatility state than in a high volatility state.

The finding that volatility asymmetry is predominantly a high volatility phenomenon has implications for the leverage effect and the volatility feedback effect. The leverage effect only matters if volatility and thus uncertainty is high, i.e. debt levels only increase the risk (volatility) of the firm if the risk (volatility) is high. Our results also explain why Linton, Whang, and Yen (2016) found only weak evidence that negative returns mat- 
ter: as their test considers the conditional distribution as a whole, the asymmetries uncovered with quantile regressions are possibly masked by the use of a single test statistic.

Similarly, the volatility feedback effect appears to be valid only in higher volatility regimes in which volatility follows an explosive process consistent with an amplifying feedback effect on returns and volatility itself (cp. the upper quantiles of the plots in Figure 2). This finding also implies that the statement "no news is good news" is only valid in low volatility regimes. In contrast, "no news" still increases volatility in a high volatility regime because there is no innovation (news) that would halt or dampen the explosive process of volatility.

The high volatility implications of the model are important for a better understanding of financial markets and volatility during periods of financial turmoil or crises. The results emphasize that volatility is not just clustered but that there is significant heterogeneity in the "clustering" with a self-nurturing explosiveness in high volatility states. This heterogeneity can in particular not be captured by a GARCH model (as the one presented in Table 1) as the autoregressive structure of the variance equation requires an average persistence parameter that holds for all levels of volatility.

\section{Volatility feedback effects}

The previous section analyzed the persistence of volatility and the effect of lagged returns on future volatility in light of the leverage effect. In contrast, this section examines the reverse direction from future volatility to lagged returns. This relationship is referred to as volatility feedback Bollerslev, Litvinova, and Tauchen (2006) and offers an alternative explanation for the asymmetric return-volatility relationship using a different causal explanation. Whilst the leverage effect is based on the idea that negative returns increase the debt to equity ratio and thus make the firm and the stock more risky, the volatility feedback effect assumes that investors demand a compensation for increased volatility reducing the current price of a firm and thus allowing a higher expected return. The dynamic structure of the effects is similar, that is, if lagged negative returns increase future volatility consistent with the leverage effect, an expected future increase in volatility decreases current prices according to the volatility feedback effect.

To study the volatility feedback effect, previous literature has to the largest extent relied on GARCH models to extract the relationship (Bae, Kimb \& Nelson, 2007; Campbell \& Hentschel, 1992; Christensen, Nielsen \& Zhu, 2010, cp.). Only more recently models based on stochastic volatility are applied to this question Bekaert and $\mathrm{Wu}(2000)$ and Bollerslev, Litvinova, and Tauchen (2006). We propose to apply the quantile autoregression framework to obtain a finer description of the volatility feedback effect for different quantiles of the return distribution as we would expect a more pronounced effect for extreme returns as opposed to returns close to zero.

In line with the analysis in the previous section ${ }^{2}$ we estimate the volatility feedback effect with a quantile regression model as follows:

$$
Q_{r_{t}}(\tau)=\theta_{0}(\tau)+\theta_{1}(\tau) E_{t}\left(R V_{t+1}\right),
$$

where $E_{t}$ denotes the conditional expectation. To implement the estimation, we replace the expected value by the observed $R V_{t+1}$.

Figure 9 summarizes the estimation results of the quantile model and a mean model (estimated with OLS) for all 20 stock market indices using realized variances. ${ }^{3}$ The upper graph displays the estimates of the intercept and the lower graph depicts the quantile-dependent estimates of $\theta_{1}$ for all $\tau$. The OLS-based mean effects are presented as horizontal (blue) lines. The dashed lines illustrate the cross-sectional dispersion of the estimators across stock market indices. The intercept varies between -2.1 and 2.6 across the quantiles, with the estimate at the median approximately zero which illustrates the distribution of returns. The black solid line in the lower graph shows that the volatility feedback effect is on average negative for lower to intermediate quantiles (up to the $60 \%$ quantile on average) and positive for the remaining upper quantiles. This means that an increase in future volatility amplifies both extreme negative returns and extreme positive returns. The OLS-based volatility feedback coefficient is negative on average which is in line with the results documented by Bollerslev and Zhou (2006) (see their Table 4) or Koopman and Uspensky (2002). 

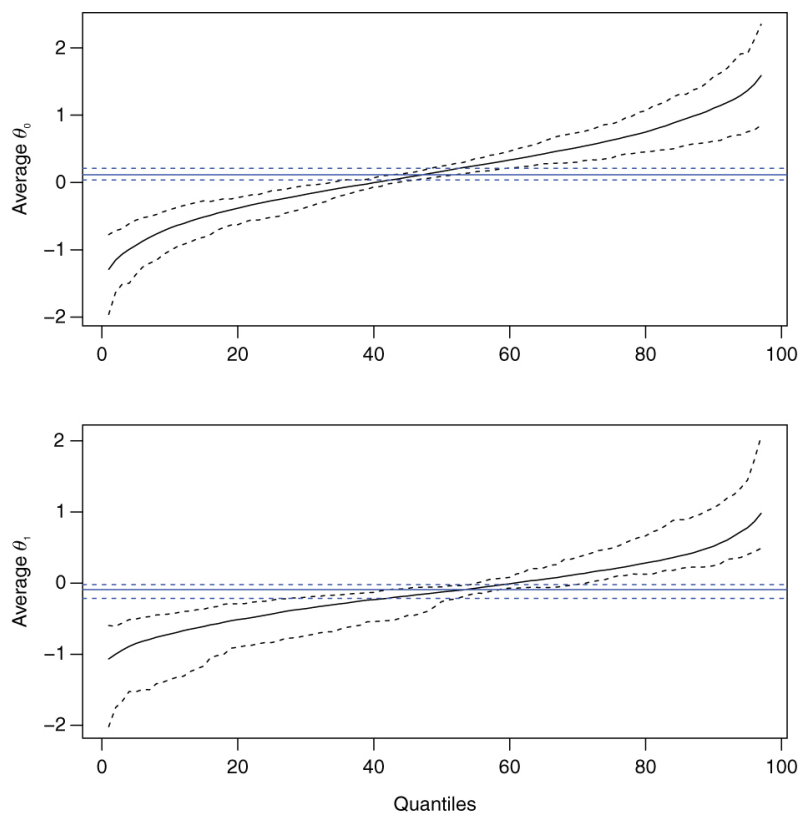

Figure 9: Volatility feedback.

The graphs depict the coefficient estimates of the quantile autoregressive model for the volatility feedback effect. The black lines are the result from the quantile regression model (the solid line is the average, the dashed lines are maximum and minimum) while the blue horizontal lines represent the outcome of a linear regression (again, the solid line is the average, the dashed lines are maximum and minimum).

The volatility feedback effect generally refers to situations in which an anticipated increase of volatility decreases the current price, i.e. increased volatility requires expected returns to rise and thus lowers the price of the asset. Hence, the positive effect for upper quantiles is a novel and possibly puzzling finding.

From a statistical point of view, the pattern documented in the second graph in Figure 9 is consistent with the volatility dynamics identified earlier in this paper: since both positive and negative returns increase future volatility, the volatility feedback effect must work in both directions as well. In other words, if positive shocks increase future volatility, anticipated increases in future volatility (as proxied by $R V_{t-1}$ cannot be exclusively associated with negative shocks. Still, the effect is estimated to be negative up to the $60 \%$ quantile on average which suggests that the largest part of the distribution of the return variable is negatively affected by the future volatility. This is in line with the finding that the OLS estimate is slightly negative.

Whilst the "classical" volatility feedback effect is first and foremost a negative return or "bad state" phenomenon the quantile-based (non-classical) effect identifies situations where investors accept higher volatility and higher prices. A potential example might be Bitcoin: the prospect of high future returns leads some investors to buy the respective asset irrespective of the present price and risk.

\section{Simulation study}

Given the large literature on jumps in volatility, the findings obtained within our quantile autoregression framework might be surprising at first glance. To ensure that the results are not a statistical artifact and to dig deeper into the relationship of jumps and quantile dependent persistence, we use a simulation study.

To construct a dataset of 5000 daily realized variance measures, we follow Barndorff-Nielsen and Shephard (2004) and Huang and Tauchen (2005) and use the following stochastic volatility jump diffusion model:

$$
d p(t)=\mu d t+\exp \left[\beta_{0}+\beta_{1} v(t)\right] d w_{p}(t)+d L_{J}(t)
$$

$$
d v(t)=\alpha_{v} v(t) d t+d w_{v}(t) .
$$

$w_{p}$ and $w_{v}$ are standard Brownian motions with correlation $\rho, v(t)$ is a stochastic volatility factor, $L_{J}(t)$ is a compound Poisson process with constant jump intensity $\lambda$ and random jump size distributed as $N\left(0, \sigma_{J}^{2}\right)$. The data generating process is implemented using the settings as in Huang and Tauchen (2005) which are represented in 
Table 2. We implement the Euler discretization based on a ten-second increment per tick which leads to 6 ticks per minute. The simulated trading day is 6.5 hours. We then compute the realized variance on a five minute interval and finally estimate the quantile autoregression model given in Equation (2) for $\tau \in[0.02,0.098]$. We omit the extreme quantiles 0.01 and 0.99 to avoid noisy estimates inflicting with our analysis. ${ }^{4}$

Table 2: Simulation settings.

\begin{tabular}{ll}
\hline$\mu$ & 0.030 \\
$\beta_{0}$ & 0.000 \\
$\beta_{1}$ & 0.125 \\
$\alpha_{v}$ & $\{-0.00137,-0.100,-0.1386\}$ \\
$\rho$ & -0.620 \\
$\lambda$ & $\{0.014,0.082,0.500,1.000\}$ \\
$\sigma_{J}$ & $\{0.00,0.50,1.00,1.50,2.00\}$ \\
\hline
\end{tabular}

The table presents the settings for the data generating process used in the simulation study.

The object of interest in the simulation is how the estimation of the persistence of realized variance, expressed first and foremost by the estimates of $\theta_{3}(\tau)$ in our QAR model is affected. As outlined in Section 3.1, there are two possible effects of jumps on the detection of persistence: first, unpredictable jumps might dominate high volatility and, hence, impede the detection of persistence in high quantiles of the realized variance. If this is the case, the estimates $\theta_{3}(\tau)$ for $\tau>0.9$ should be close to zero as no persistence can be detected in this case. If, on the other hand, jumps induce non-stationarity, we should observe $\theta_{3}(\tau) \approx 1$ for that proportion of the daily RV observations that is "contaminated" with jumps. This effect should be more pronounced as the jump intensity $\lambda$ or the jump size $\sigma_{J}$ increase. To investigate these issues, we report averages of the $\theta_{3}(\tau)$ estimates for low (0.02-0.1), intermediate (0.45-0.55), and high quantiles (0.90-0.98).

Table 3 holds the results for a mildly mean-reverting volatility process $\left(\alpha_{v}=-0.1\right)$ and presents the average coefficient estimates for the stock markets for comparison. The bigger picture that these results suggest is that moderate jumps do not inhibit the detection of persistence and persistence is higher in high quantiles. As jumps become more important, either through jump intensity $\lambda$ or jump size $\sigma_{J}$, they overshadow the persistence. ${ }^{5}$

Table 3: Simulation results.

\begin{tabular}{|c|c|c|c|c|}
\hline$\lambda$ & $\sigma_{J}$ & Low & Intermediate & High \\
\hline 0.014 & 0 & 0.4493 & 0.6874 & 1.0543 \\
\hline 0.014 & 0.5 & 0.4281 & 0.6879 & 1.0521 \\
\hline 0.014 & 1 & 0.3746 & 0.6869 & 1.0493 \\
\hline 0.014 & 1.5 & 0.3067 & 0.6779 & 1.0402 \\
\hline 0.014 & 2 & 0.2667 & 0.6706 & 1.0428 \\
\hline 0.082 & 0 & 0.4487 & 0.6904 & 1.0543 \\
\hline 0.082 & 0.5 & 0.3065 & 0.6700 & 1.0445 \\
\hline 0.082 & 1 & 0.1085 & 0.6474 & 1.0392 \\
\hline 0.082 & 1.5 & 0.0283 & 0.5825 & 1.0119 \\
\hline 0.082 & 2 & 0.0141 & 0.4469 & 0.9591 \\
\hline 0.5 & 0 & 0.4512 & 0.6911 & 1.0473 \\
\hline 0.5 & 0.5 & 0.0226 & 0.5689 & 0.9842 \\
\hline 0.5 & 1 & -0.0001 & 0.2041 & 0.7564 \\
\hline 0.5 & 1.5 & -0.0001 & 0.0026 & 0.4056 \\
\hline 0.5 & 2 & 0.0000 & 0.0001 & 0.1267 \\
\hline 1 & 0 & 0.4506 & 0.6883 & 1.0519 \\
\hline 1 & 0.5 & 0.0049 & 0.4300 & 0.7967 \\
\hline 1 & 1 & -0.0002 & 0.0073 & 0.3166 \\
\hline 1 & 1.5 & -0.0001 & 0.0002 & 0.0768 \\
\hline 1 & 2 & 0.0000 & -0.0001 & 0.0488 \\
\hline Stock markets & & 0.0887 & 0.2771 & 0.9635 \\
\hline
\end{tabular}

The table presents the results of the simulation study for different settings of the the jump intensity $\lambda$ and the jump size variation $\sigma_{I}$ for a mildly mean reverting volatility process $\left(\alpha_{v}=-0.1\right)$. Columns 3 to 5 report the average estimates $\theta_{3}(\tau)$ for low $(\tau$ from 0.02 to 0.1$)$, intermediate $(0.45$ to 0.55$)$, and high quantiles $(0.90$ to 0.98$)$. The last line presents the results based on the sample of stock market indices for comparison. 
In more detail the results are as follows. If the volatility process exhibits mild mean reversion $\left(\alpha_{\mathrm{v}}=-0.1\right)$, we obtain results that are similar to the empirical findings documented in Section 3 if jump intensity is low and jump size is high $\left(\lambda=0.082\right.$ and $\left.\sigma_{J}=2\right)$ or if jump intensity is medium and jump size is small $\left(\lambda=0.5\right.$ and $\sigma_{J}=$ 0.5). As both features are connected, obtaining similar results for close settings seems plausible.

For a given jump intensity, we find that as jump size increases, first the low quantile estimates are affected. For low jump intensity $(\lambda=0.014)$ the persistence in the upper quantiles is basically not affected at all. Only as jump intensity increases, the high quantile estimates are also reduced, indicating that at some stage, jumps dominate the process which is therefore not predictable any longer. The extreme case is documented for $\lambda=1$. Here, already small jumps in the return equation $\left(\sigma_{I}=0.5\right)$ superpose the persistence of the volatility equation in the realized variance. For higher values of $\sigma_{J}$, persistence cannot be estimated from the realized variance any longer, the quantile regression estimates are virtually zero.

In a second step, we also varied $\alpha_{v}$ (detailed results are provided in the appendix). We find that as the absolute value of $\alpha_{v}$ increases (i.e. stronger mean reversion), the overall persistence is reduced. For example set $\lambda=$ 0.014 and $\sigma_{J}=0.5$. In this case, the average quantile estimates are $0.50,0.78$, and 1.21 for the low, intermediate and high quantiles, respectively, if $\alpha_{\mathrm{v}}=-0.00137$. This setting is highly persistent as documented by the high QAR estimates which increase from low to high quantiles as we would have expected. Now as the mean reversion increases $\left(\alpha_{\mathrm{v}}=-0.1\right)$, the persistence is reduced and we find the respective average quantile estimates to be $0.43,0.69$, and 1.05 (see Table 3 ). If the mean reversion in the volatility process is even stronger $\left(\alpha_{\mathrm{v}}=-1.386\right)$, the QAR estimates documenting persistence reduce to $0.06,0.13$, and 0.16 , respectively.

The way jumps impact on the detection of persistence, however, is not altered. It still holds that low jump intensity and low jump size lead to qualitatively similar results as if there were no jumps. Only when the jump intensity is high $(\lambda \geq 0.5)$ and/or the jump size is high $\left(\sigma_{J} \geq 1\right)$, jumps becloud the detection of the persistence. Of course, the stronger the persistence, i.e.the lower $\left|\alpha_{v}\right|$, the less is the impact of jumps felt in estimating persistence.

\section{Summary and concluding remarks}

This paper proposes to rethink the stylized facts of volatility clustering and the asymmetric volatility effect. We use quantile regression to analyze the persistence of realized variance and the predictive asymmetry of positive and negative shocks conditional on the state or regime of volatility. We find that low volatility states are very different from high volatility states regarding both the persistence and the asymmetry of volatility. Only high volatility states exhibit pronounced persistence of volatility - even displaying explosive behavior. Similarly, the predictive asymmetry of volatility, i.e. negative shocks increase future volatility by more than positive shocks, is only a high volatility phenomenon and there is no asymmetry in low volatility regimes. The explosive behavior in high quantiles is a phenomenon that has not been documented so far.

Our findings also explain the weak empirical evidence for the "leverage effect" as most studies focus on the average effect and do not condition on the states of volatility. We argue that the results are economically plausible: a firm's leverage will be more important in a high volatility (high risk) regime than in a low volatility environment and volatility feedback effects will be larger and thus more important in high volatility regimes than in low volatility regimes. Finally, if volatility is high and follows an explosive process, "no news" is not "good news" as there is no break in the explosive volatility process. The special role of high volatility regimes with its explosive processes also suggest that financial markets are unstable in high volatility regimes with strong implications for the financial system architecture and policy makers.

In a nutshell, the paper proposes to use an alternative estimation framework for volatility that could also be used, for example, to analyze regime-specific volatility spillovers. Given the large and growing literature on volatility spillovers and contagion, there is ample opportunity for future research.

\section{Appendix}

Table 4: Full simulation results.

\begin{tabular}{lrrrrr}
\hline $\boldsymbol{\alpha}_{v}$ & $\boldsymbol{\lambda}$ & $\sigma_{J}$ & Low & Intermediate & High \\
\hline-0.00137 & 0.014 & 0 & 0.5022 & 0.7715 & 1.2034 \\
-0.00137 & 0.014 & 0.5 & 0.5011 & 0.7757 & 1.2089 \\
-0.00137 & 0.014 & 1 & 0.4772 & 0.7716 & 1.2153
\end{tabular}




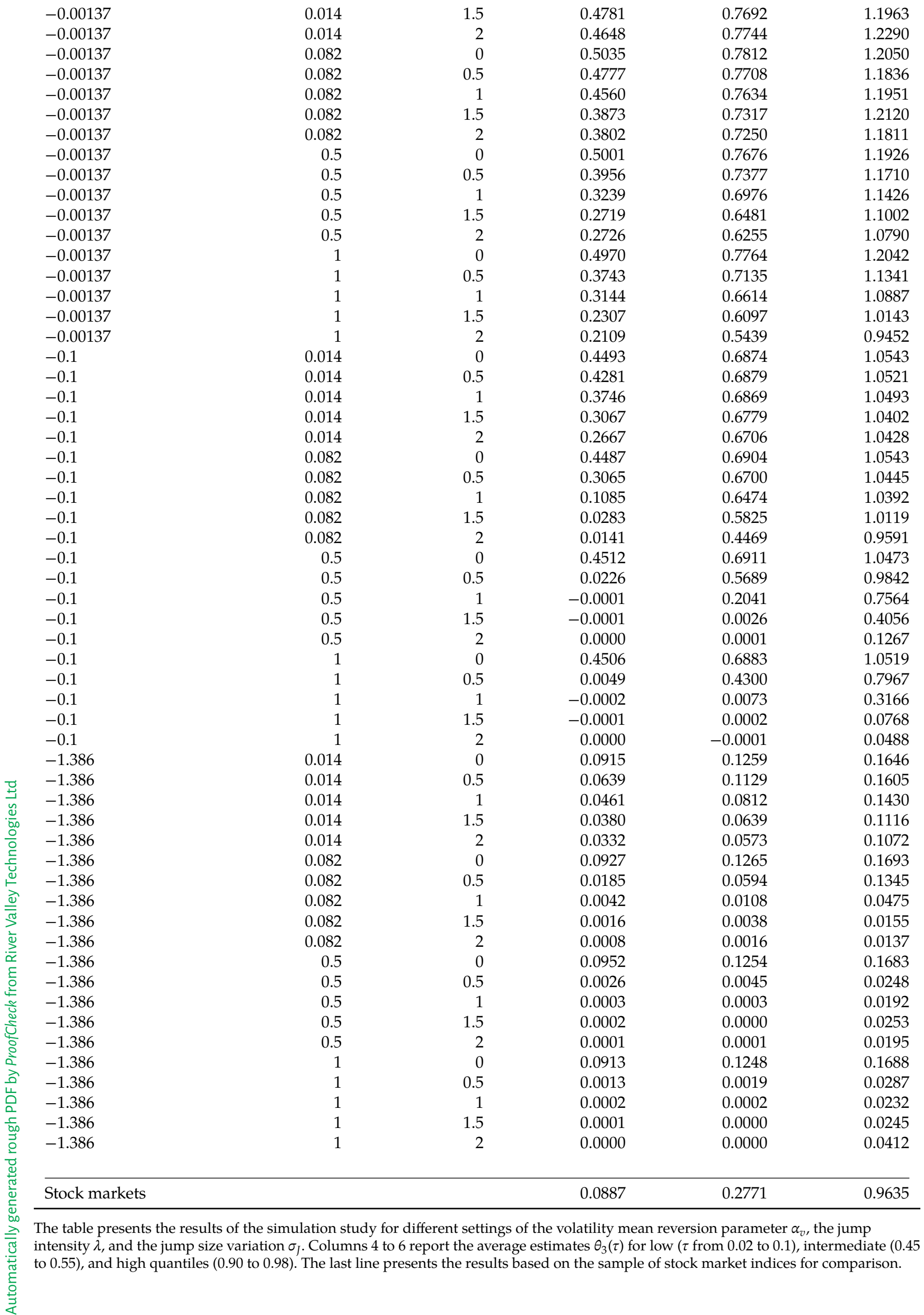




\section{Notes}

1 Sub-sampling and estimating the TGARCH or RV model for a restricted sample might, to some extent, alleviate the issue of confining the dynamics to one single estimator. However, doing so creates two new issues: determining the appropriate time period for the sub-sample, and a reduction of the number of available observations until the sample is too small for a reliable estimation.

2 Note that the model structure differs from the contemporaneous model structure used in Bollerslev, Litvinova, and Tauchen (2006) but the estimation results also hold for a contemporaneous model.

3 As a robustness check we also implement the model for volatilities $R V_{t}^{0.5}$ instead of $R V_{t}$ and find similar results.

4 We also implemented the simulation for 15,000 daily RV observations and find that the (unreported) results are qualitatively similar to the results obtained with 5000 daily observations which leads us to conclude that a sample of the size as used in the empirical analysis is sufficient to reliably estimate also extreme quantiles in this particular QAR application.

5 In order to exclude the possibility that the asymmetric patterns are purely due to measurement error in realized volatility, we also estimated the QAR model using the simulated daily variance directly. While the coefficient estimates are not identical, the dispersion pattern and the conclusions with respect to the jump size still hold.

\section{References}

Andersen, T. G., T. Bollerslev, F. X. Diebold, and P. Labys. 2003. “Modeling and Forecasting Realized Volatility." Econometrica 71: 529-626. Badshah, I. U. 2013. “Quantile Regression Analysis of Asymmetric Return-Volatility Relation." Journal of Futures Markets 33: 235-265.

Bae, J., C.-J. Kimb, and C. R. Nelson. 2007. "Why are Stock Returns and Volatility Negatively Correlated?" Journal of Empirical Finance 14: 41-58. Barndorff-Nielsen, O. E., and N. Shephard. 2004. "Power and Bipower Variation with Stochastic Volatility and Jumps." Journal of Financial Econometrics 2: 1-48.

Baur, D. G., and T. Dimpfl. 2016. “Inter-quantile Ranges and Volatility of Financial Data.” Available at SSRN: https://ssrn.com/abstract=2835951.

Baur, D. G., T. Dimpfl, and R. C. Jung. 2012. “Stock Return Autocorrelations Revisited: A Quantile Regression Approach.” Journal of Empirical Finance 19: 254-265.

Bekaert, G., and G. Wu. 2000. "Asymmetric Volatility and Risk in Equity Markets." Review of Financial Studies 13: 1-42.

Bekaert, G., E. Engstrom, and A. Ermolov. 2015. “Bad Environments, Cood Environments: A Non-Caussian Asymmetric Volatility Model.” Journal of Econometrics 186: 258-275.

Bollerslev, T., and H. Zhou. 2006. “Volatility Puzzles: A Simple Framework for Gauging Return-Volatility Regressions." Journal of Econometrics 131: 123-150.

Bollerslev, T., J. Litvinova, and G. Tauchen. 2006. “Leverage and Volatility Feedback Effects in High-Frequency Data." Journal of Financial Econometrics 4: 353-384.

Campbell, J. Y., and R. J. Shiller. 1988. “The Dividend-Price Ratio and Expectations of Future Dividends and Discount Factors." Review of Financial Studies 1: 195-228.

Campbell, J. Y., and L. Hentschel. 1992. "No News is Cood News: An Asymmetric Model of Changing Volatility in Stock Returns." Journal of Financial Economics 31: 281-318.

Christensen, B. J., M. O. Nielsen, and J. Zhu. 2010. "Long Memory in Stock Market Volatility and the Volatility-in-Mean Effect: The FIECARCHM Model." Journal of Empirical Finance 17: 460-470.

Corsi, F. 2009. “A Simple Approximate Long-Memory Model of Realized Volatility." Journal of Financial Econometrics 7: 174-196.

Corsi, F., and R. Renò. 2012. "Discrete-Time Volatility Forecasting with Persistent Leverage Effect and the Link with Continuous-Time Volatility Modeling." Journal of Business and Economic Statistics 30: 368-380.

Ederington, L. H., and W. Guan. 2010. “How Asymmetric is U.S. Stock Market Volatility?” Journal of Financial Markets 13: $225-248$.

Engle, R. F., and V. K. Ng. 1993. "Measuring and Testing the Impact of News on Volatility." The Journal of Finance 48: 1749-1778.

French, K. R., G. W. Schwert, and R. F. Stambaugh. 1987. “Expected Stock Returns and Volatility.” Journal of Financial Economics 19: 3-29.

Closten, L. R., R. Jagannathan, and D. E. Runkle. 1993. “On the Relation Between the Expected Value and the Volatility of the Nominal Excess Return on Stocks." The Journal of Finance 48: 1779-1801.

Haas, M., J. Krause, M. S. Paolella, and S. C. Steude. 2013. "Time-Varying Mixture Garch Models and Asymmetric Volatility." North American Journal of Economics and Finance 26: 602-623.

Heber, G., A. Lunde, N. Shephard, and K. Sheppard. 2009. “Oxford-Man Institute's Realized Library.” Technical report, Oxford-Man Institute, University of Oxford, library Version 0.2 .

Huang, X., and C. Tauchen. 2005. “The Relative Contribution of Jumps to Total Price Variance.” Journal of Financial Econometrics 3: 456-499. Koenker, R., and G. Bassett, Jr. 1978. “Regression Quantiles.” Econometrica 46: 33-50.

Koenker, R., and Z. Xiao. 2006. “Quantile Autoregression.” Journal of the American Statistical Association 101: 980-990.

Koopman, S. J., and E. H. Uspensky. 2002. "The Stochastic Volatility in Mean Model: Empirical Evidence from International Stock Markets." Journal of Applied Econometrics 17: 667-689.

Linton, O., Y.-J. Whang, and Y.-M. Yen. 2016. “A Nonparametric Test of a Strong Leverage Hypothesis." Journal of Econometrics 194: 153-186.

Nelson, D. B. 1991. “Conditional Heteroskedasticity in Asset Returns: A New Approach.” Econometrica 59: 347-370.

Park, B.-J. 2011. "Asymmetric Herding as a Source of Asymmetric Return Volatility." Journal of Banking \& Finance 35: 2657-2665.

Patton, A. J., and K. Sheppard. 2015. "Cood Volatility, Bad Volatility: Signed Jumps and the Persistence of Volatility." Review of Economics and Statistics 97: 683-697.

Schwarz, G. 1978. “Estimating the Dimension of a Model." Annals of Statistics 6: 461-464. 
Shephard, N., and K. Sheppard. 2009. "Realising the Future: Forecasting with High Frequency Based Volatility (Heavy) Models." University of Oxford Department of Economics Discussion Paper Series.

Talpsepp, T., and M. O. Rieger. 2010. “Explaining Asymmetric Volatility Around the World.” Journal of Empirical Finance 17: 938-956.

Wu, C. 2001. "The Determinants of Asymmetric Volatility." Review of Financial Studies 14: 837-859.

Zhu, H., Y. Guo, W. You, and Y. Xu. 2016. "The Heterogeneity Dependence Between Crude Oil Price Changes and Industry Stock Market Returns in China: Evidence from a Quantile Regression Approach." Energy Economics 55: 30-41.

Zhu, H.-M., Z. Li, W. You, and Z. Zeng. 2015. “Revisiting the Asymmetric Dynamic Dependence of Stock Returns: Evidence from a Quantile Autoregression Model." International Review of Financial Analysis 40: 142-153.

Supplemental Material: The online version of this article offers supplementary material (https://doi.org/10.1515/snde-2017-0020). 